\title{
COVID-19 and HIV: Case reports of 2 co-infected patients with different disease courses
}

\author{
RICARDO WESLEY ALBERCA, VALERIA AOKI and MARIA NOTOMI SATO \\ Laboratory of Dermatology and Immunodeficiencies, LIM-56, Department of Dermatology, School of Medicine \\ and Institute of Tropical Medicine of São Paulo, University of São Paulo, São Paulo, SP 05403-000, Brazil
}

Received October 20, 2020; Accepted November 25, 2020

DOI: $10.3892 /$ wasj.2020.75

\begin{abstract}
The ongoing coronavirus disease 2019 (COVID-19) pandemic has affected thousands of patients and thousands have also succumbed to the disease. The literature reports risk factors, such as obesity, diabetes and an older age. However, the association between immunosuppression caused by diseases, such as human immunodeficiency virus (HIV), and COVID-19 has been rarely reported. In the present study, the cases of 2 HIV-infected individuals (aged 48 and 49 years) with COVID-19 symptoms are presented. At 4 days following the onset of COVID-19 symptoms, the patients tested positive for the syndrome due to coronavirus-2 (SARS-CoV-2) infection by RT-PCR. The patients were hospitalized in the general ward of a tertiary university hospital for COVID-19 patients (Hospital das Clinicas from the Medical School of the University of São Paulo), until discharge. Both patients were age-matched HIV-positive patients and presented different SARS-CoV-2 infection courses. In summary, the findings reported herein highlight that HIV-infected individuals may be a high-risk group for developing severe COVID-19, particularly in association with other risk factors, such as obesity, type 2 diabetes mellitus and systemic arterial hypertension.
\end{abstract}

\section{Introduction}

The human immunodeficiency virus (HIV) is a lentivirus within the family of Retroviridae, subfamily Orthoretrovirinae, that enters the body via mucous membranes, injured skin or by parenteral inoculation. This infection can progress with the marked reduction in CD4 cell numbers $(<300 / \mu \mathrm{l})$

Correspondence to: Dr Ricardo Wesley Alberca, Laboratory of Dermatology and Immunodeficiencies, LIM-56, Department of Dermatology, School of Medicine and Institute of Tropical Medicine of São Paulo, University of São Paulo, Av. Dr. Enéas Carvalho de Aguiar 470, São Paulo, SP 05403-000, Brazil

E-mail: ricardowesley@usp.br

Key words: SARS-CoV-2, HIV, COVID-19, infection and the immune response to respiratory microorganisms is suppressed (1).

The deleterious effects of HIV are controlled with anti-retroviral drugs (2). Several antiviral drugs, such as lopinavir, remdesivir and umifenovir, have been tested without marked success for coronavirus disease 2019 (COVID-19) (3). COVID-19 infection, characterized by severe acute respiratory syndrome due to coronavirus-2 (SARS-CoV-2), can lead to severe respiratory illness, with neutrophilia and lymphopenia, and an increase in the levels of inflammatory markers, such as C-reactive protein, urea, creatinine and D-dimer (4).

Due to the ongoing COVID-19 pandemic, the investigation of comorbidities (5-7) or concomitant infection with COVID-19 are extremely relevant $(8,9)$. To date, there is no current standard treatment available, and new treatments are currently under investigation, particularly in higher-risk populations (10-12). Therefore, in order to evaluate the influence of HIV infection on COVID-19, in the present study, the cases of 2 female patients of a similar age (48 and 49 years) are presented, who were followed-up daily, from the time of hospitalization to SARS-CoV2 clearance.

\section{Case reports}

Patients were recruited at the Hospital das Clínicas of the University of São Paulo (HCFMUSP), a public tertiary university hospital with a special ward for COVID-19 patients. Patients were selected on the bases of previous HIV infection and recent SARS-CoV-2 infection. The diagnosis of SARS-CoV-2 infection was confirmed by the nasopharyngeal detection of SARS-CoV-2 RNA by reverse transcription-polymerase chain reaction (RT-PCR). Patients tested negative for influenza and respiratory syncytial virus. Informed consent was obtained from both patients. The present study was approved by the Ethics Committee of HCFMUSP (no. 30800520.7.0000.0068-2020), and was performed out in conformity with the 2013 revision of the Declaration of Helsinki.

Laboratory analysis was performed at the Central Laboratory of HCFMUSP. Laboratory analysis included the following: complete blood counts (CBCs), and alanine aminotransferase (ALT), aspartate aminotransferase (AST), creatinine, glucose, C-reactive protein, total protein (albumin and globulin) and urea levels, prothrombin time and activated partial thromboplastin time. 

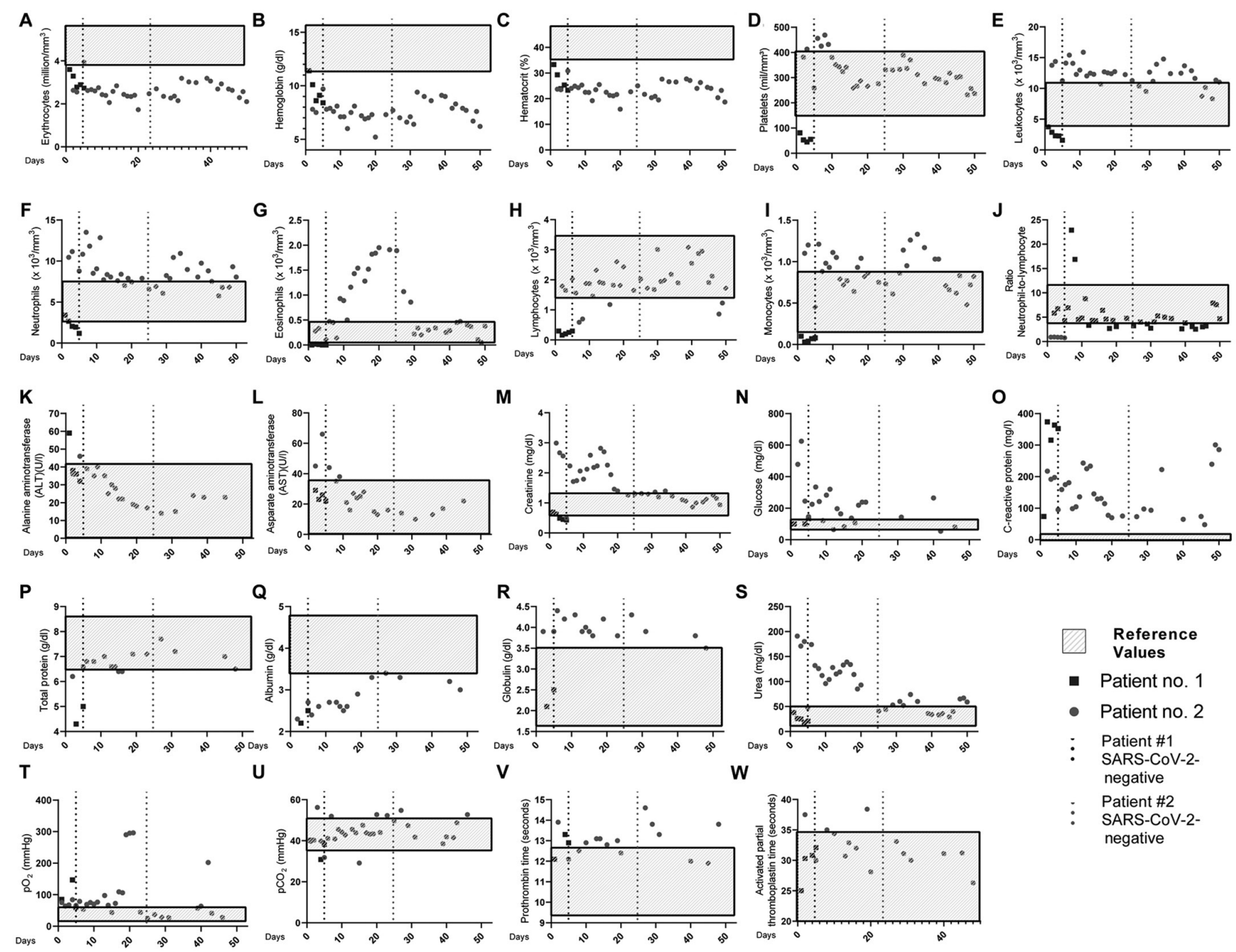

Figure 1. Daily clinical features of the 2 patients. Blood levels of (A) erythrocytes, (B) hemoglobin, (C) hematocrit, (D) platelets, (E) leukocytes, (F) neutrophils, (G) eosinophils, (H) lymphocytes, (I) monocytes, (J) neutrophil-to-lymphocyte ratio, (K) ALT, (L) AST, (M) creatinine, (N) glucose, (O) C-reactive protein, (P) total protein, (Q) albumin, (R) globulin, (S) urea, (T) $\mathrm{pO}_{2}$, (U) $\mathrm{pCO}_{2}$, (V) prothrombin time, (W) activated partial thromboplastin time. The light grey rectangular boxes represent reference values; black squares represent patient no. 1; closed grey circles represent patient no. 2; dotted lines represent the date the patients were cleared of SARS-CoV-2 infection.

Patient no. 1. A female, non-pregnant, HIV-positive, 48-year-old patient, weighing $75 \mathrm{~kg}$, with blood glucose levels of $100 \mathrm{mg} / \mathrm{dl}$, attended the hospital on May 21, 2020, with a 5-day history of COVID-19-associated symptoms that had begun on May 16, 2020. The diagnosis of COVID-19 was confirmed on May 21, 2020. Patient no. 1 used Tenofovir, Lamivudi, Dolutegravir and Topotecan for the control of HIV on a daily basis; HIV-infection date was not available.

For COVID-19 infection, the patient received the following treatment: Oseltamivir (75 mg; May 23 to May 26), Ceftriaxone (2 g; May 23 to May 26), Clexane (40 mg; May 23), Ceftriaxone ( 2 g; May 22), Prednisone (40 mg; May 26), Azythromycin $(500 \mathrm{mg}$; May 22 to May 26) and Levofloxacin $(750 \mathrm{mg}$; May 26).

As depicted in Fig. 1 (black squares), patient no. 1 exhibited reduced erythrocytes, hemoglobin, hematocrit and platelet levels on the first day of hospitalization. Of note, the patient presented normal leukocyte, lymphocyte and neutrophil numbers. The only increased COVID-19-associated severity marker was C-reactive protein $(73.7 \mathrm{mg} / \mathrm{l})$, present at day 1 (4).

The patient was cleared of SARS-CoV-2 infection on day 10 after the beginning of the symptoms ( 5 days after the diagnosis of COVID-19). The patient was cured of COVID-19 and released from the hospital on May 26, 2020.

Patient no. 2. A female non-pregnant, 49-year-old patient, weighing $90 \mathrm{~kg}$, obese, with a body mass index (BMI) of 33.05 and blood glucose levels of $478 \mathrm{mg} / \mathrm{dl}$, attended the hospital on May 1, 2020 with a 4-day history of COVID-19-associated symptoms. COVID-19 was confirmed on May 1, 2020.

The patient's history included the following: Type 2 diabetes mellitus, obesity, 2 previous heart attacks, HIV positivity since 2009 , and the regular daily usage of quetiapine, captopril and furosemide, omeprazole and enoxaparin, and darunavir for the control of HIV. No HIV viral load was detectable.

During the COVID-19 infection, the patient underwent a series of drug treatments: Ampicyllin (2 g; May 4 to May 12), Polymyxin B (1000000 Units; May 12 to May 14), Meropenem (1 g; May 11 to May 19) and Vancomyicin (1 g; May 12 to May 19), Enoxaparin (80 mg) and Ceftriaxone (2 g; May 21 to May 26), Azythromycin (500 mg; May 21 to May 25), Oseltamivir (75 mg; May 22).

As depicted in Fig. 1 (closed grey circles), patient no. 2 exhibited reduced erythrocyte, hemoglobin and hematocrit 
levels. Of note, the patient exhibited an increase in neutrophil numbers, but not in the neutrophil-to-lymphocyte ratio (13). Other laboratory features which were above the reference values were AST, creatinine, C-reactive protein and urea (4).

The patient was cleared of SARS-CoV-2 infection on day 29 after the beginning of the symptoms (25 days after the diagnosis of COVID-19), but remained in the hospital due to alterations in laboratory data. Notably, 10 days prior to the SARS-CoV-2 infection, the eosinophil count began to increase, peaking at 1 day prior to SARS-CoV-2 clearance, and abruptly decreasing to regular levels over the following days. Blood glucose and creatinine levels also reached normal levels almost concomitantly to SARS-CoV-2 clearance (Fig. 1; closed grey circles).

Patient no. 2 was cured of COVID-19 and released from the special ward for COVID-19 patients and being transferred to the general ward on June 25 and later discharged on June 30, 2020 .

\section{Discussion}

Both patients presented herein had a similar age and were HIV-positive with no detectable viral load. Even though they had SARS-CoV-2 infection, the general clinical features were markedly distinct between them. Patient no. 1 had leucopenia and a low neutrophil-to-lymphocyte ratio, a hallmark of severity in COVID-19 $(13,14)$. Patient no. 2 exhibited an increase in leukocyte and neutrophil numbers, but maintained a normal level of the neutrophil-to-lymphocyte ratio. A common feature between both patients was the elevated c-reactive protein levels and low levels of serum albumin, both previously associated with severity in COVID-19 (15).

A distinguishing feature between both patients was blood glucose levels: patient no. 1 evolved with normal levels during hospitalization, while patient no. 2 exhibited elevated blood glucose levels during SARS-CoV-2-infection, which and returned to close to normal ranges following the clearance of SARS-CoV-2. A recent study described that high glucose levels may facilitate SARS-CoV-2 replication, and therefore promote and exacerbate the inflammatory response by monocytes (16). Of note, patient no. 2 also presented an increase in circulating monocyte numbers. Monocytes have been also implicated in the production of IL- 6 and the induction of the cytokine storm in COVID-19, further increasing IL-6 production in intensive care unit patients (17).

Although previous research has not identified the mechanisms through which eosinophils may contribute to COVID-19 resolution, it has been hypothesized that an increase in eosinophil numbers may be an indicator of COVID-19 improvement (18). These findings are in accordance with the present findings, revealing an increase in the eosinophil count previous to SARS-CoV-2 clearance in patient no. 2 .

A striking difference between the 2 patients was the SARS-CoV-2 clearance time: This was 10 days for patient no. 1 (5 days after hospitalization) and 29 days for patient no. 2 (25 days after hospitalization). A previous case report on a patient with HIV identified a delayed immune response against SARS-CoV-2, with detectable blood IgM identified 60 days following infection (19). A larger cohort study also verified that almost one-third of HIV-positive patients remained positive for SARS-CoV-2 after 40 days of the initiation of symptoms (20).

Härter et al identified that among HIV-positive fatalities, other comorbidities than HIV infection may present a larger role in SARS-CoV-2 infection (2). In the present case report, this appears to be true, as both patients exhibited an undetectable HIV viral load and a similar CD4/CD8 cell count. A hallmark difference between the 2 patients was the presence of comorbidities other than HIV. Patient no. 1 had no comorbidities, while patient no. 2 had systemic arterial hypertension (SAH), type 2 diabetes mellitus and obesity (body mass index, 33.05).

HIV infection also predisposes individuals to the development of type 2 diabetes mellitus (21), via alterations in adipocyte and T-cell metabolism, increased lipolysis, hyperglycemia, hypertriglyceridemia and hepatic steatosis (21). Obesity also increases T-cell activation and pro-inflammatory cytokine production in consequence of the caloric surplus and an increase in adipose tissue inflammation. Therefore, HIV and obesity may play a synergic role, impairing the anti-SARS-CoV-2 immune response (22).

Patient no. 2 also presented SAH, a risk factor for mortality in COVID-19 $(4,23)$. It is noteworthy that there is no consensus as to whether HIV or anti-viral treatment may influence SAH development; however, obesity, type 2 diabetes mellitus and SAH are associated, as approximately $80 \%$ of patients with type 2 diabetes develop SAH (24).

A recent retrospective study identified no differences in the infection rate and severity on SARS-CoV-2 infection regarding HIV infection (25); nevertheless, the effects of COVID-19 on patients with acquired immunodeficiency syndrome (AIDS) warrant further in-depth investigations (26). Overall, the present case report study reinforces a potential role for other comorbidities, rather than HIV infection as regards SARS-CoV-2 infection outcomes.

\section{Acknowledgements}

Not applicable.

\section{Funding}

The present study was funded by the Fundação de Amparo à Pesquisa do Estado de São Paulo (FAPESP; grant nos. 19/02679-7 and 17/18199-9).

\section{Availability of data and materials}

Data sharing is not applicable to this article, as no datasets were generated or analyzed during the current study.

\section{Authors' contributions}

RWA conceived and designed the study, and performed the data analysis and interpretation. VA conceived and designed the study, and performed the data analysis and interpretation. MNS performed the data analysis and interpretation. All the authors contributed to the writing and final approval of the manuscript and agree to be accountable for all aspects of the research in ensuring that the accuracy or integrity of any part of the work are appropriately investigated and resolved. 


\section{Ethics approval and consent to participate}

The present study was approved by the Ethics Committee of Hospital das Clínicas from the University of São Paulo (HCFMUSP; no. 30800520.7.0000.0068-2020), and was performed out in conformity with the 2013 revision of the Declaration of Helsinki. Informed consent was obtained from both patients.

\section{Patient consent for publication}

Informed consent was obtained from both patients.

\section{Competing interests}

The authors declare that they have no competing interests,

\section{References}

1. Jambo KC, Sepako E, Fullerton DG, Mzinza D, Glennie S, Wright AK, Heyderman RS and Gordon SB: Bronchoalveolar $\mathrm{CD} 4^{+} \mathrm{T}$ cell responses to respiratory antigens are impaired in HIV-infected adults. Thorax 66: 375-382, 2011.

2. Härter G, Spinner CD, Roider J, Bickel M, Krznaric I, Grunwald S, Schabaz F, Gillor D, Postel N, Mueller MC, et al: COVID-19 in people living with human immunodeficiency virus: A case series of 33 patients. Infection 48: 681-686, 2020.

3. Hussain N, Yoganathan A, Hewage S, Alom S and Harky A: The effect of antivirals on COVID-19: A systematic review. Expert Rev Anti Infect Ther: Oct 20, 2020 (Epub ahead of print).

4. Chen G, Wu D, Guo W, Cao Y, Huang D, Wang H, Wang T, Zhang $\mathrm{X}$, Chen $\mathrm{H}$, Yu H, et al: Clinical and immunological features of severe and moderate coronavirus disease 2019. J Clin Invest 130 2620-2629, 2020.

5. Branco ACCC, Sato MN and Alberca RW: The possible dual role of the ACE2 receptor in asthma and coronavirus (SARS-CoV2) infection. Front Cell Infect Microbiol 10: 550571, 2020.

6. Alberca RW, Pereira NZ, Oliveira LMDS, Gozzi-Silva SC and Sato MN: Pregnancy, viral infection, and COVID-19. Front Immunol 11: 1672, 2020.

7. Alberca RW: Asthma endotypes and COVID-19. J Asthma: Oct 1, 2020 (Epub ahead of print).

8. Mirzaei H, McFarland W, Karamouzian M and Sharifi $H$ : COVID-19 among people living with HIV: a systematic review. AIDS Behav: Jul 30, 2020 (Epub ahead of print).

9. Alberca RW, Yendo TM, Leuzzi Ramos YÁ, Fernandes IG, Oliveira LM, Teixeira FME, Beserra DR, de Oliveira EA, Gozzi-Silva SC, Andrade MMS, et al: Case report: COVID-19 and Chagas disease in Two coinfected patients. Am J Trop Med Hyg: Oct 6, 2020 (Epub ahead of print).

10. Marino A, Pampaloni A, Scuderi D, Cosentino F, Moscatt V, Ceccarelli M, Gussio M, Celesia BM, Bruno R, Borraccino S, Borraccino $\mathrm{S}$, et al: High-flow nasal cannula oxygenation and tocilizumab administration in patients critically ill with COVID-19: A report of three cases and a literature review. World Acad Sci J 2: 23, 2020

11. Chen H, Zhang L, He Z, Wang D, Liu L, Xhang W, Chen T, Dai Z, Han $\mathrm{Z}$ and Chen M: Systemic administration of human umbilical cord-derived mesenchymal stem cells effectively ameliorates the outcomes of a critically ill elderly patient with COVID-19 with multiple comorbidities: A case report. World Acad Sci J 2: 29, 2020 .
12. Alberca RW, Teixeira FME, Beserra DR, de Oliveira EA, Andrade MMS, Pietrobon AJ and Sato MN: Perspective: The Potential Effects of Naringenin in COVID-19. Front Immunol 11: $570919,2020$.

13. Liu Y, Du X, Chen J, Jin Y, Peng L, Wang HHX, Luo M, Chen L and Zhao Y: Neutrophil-to-lymphocyte ratio as an independent risk factor for mortality in hospitalized patients with COVID-19. J Infect 81: e6-e12, 2020.

14. Alberca RW, Andrade MMS, Branco ACCC, Pietrobon AJ, Pereira NZ, Fernandes IG, Oliveira LM, Teixeira FME, Beserra DR, de Oliveira EA, et al: Frequencies of CD33+CD11b+ HLA-DR-CD14-CD66b+ and CD33+CD11b+HLA-DR-CD14+ CD66b- cells in peripheral blood as severity immune biomarkers in COVID-19. Front Med (Lausanne) 7: 580677, 2020.

15. de la Rica R, Borges M, Aranda M, Del Castillo A, Socias A, Payeras A, Rialp G, Socias L, Masmiquel L and GonzalezFreire M: Low albumin levels are associated with poorer outcomes in a case series of COVID-19 patients in Spain: a retrospective cohort study. Microorganisms 8: 1106, 2020.

16. Codo AC, Davanzo GG, Monteiro LB, de Souza GF, Muraro SP, Virgilio-da-Silva JV, Prodonoff JS, Carregari VC, de Biagi Junior CAO, Crunfli F, et al: Elevated glucose levels favor SARS-CoV-2 infection and monocyte response through a HIF-1 $\alpha /$ glycolysis dependent axis. Cell Metab 32: 498-499, 2020.

17. Zhou Y, Fu B, Zheng X, Wang D, Zhao C, Qi Y, Sun R, Tian Z, $\mathrm{Xu} \mathrm{X}$ and Wei $\mathrm{H}$ : Pathogenic T cells and inflammatory monocytes incite inflammatory storm in severe COVID-19 patients. Natl Sci Rev 7: 998-1002, 2020.

18. Liu F, Xu A, Zhang Y, Xuan W, Yan T, Pan K, Yu W and Zhang J: Patients of COVID-19 may benefit from sustained Lopinavir-combined regimen and the increase of Eosinophil may predict the outcome of COVID-19 progression. Int J Infect Dis 95: 183-191, 2020.

19. Wang M, Luo L, Bu H and Xia H: One case of coronavirus disease 2019 (COVID-19) in a patient co-infected by HIV with a low CD4+ T-cell count. Int J Infect Dis 96: 148-150, 2020.

20. Vizcarra P, Pérez-Elías MJ, Quereda C, Moreno A, Vivancos MJ, Dronda F, Casado JL, Moreno S, Pérez-Elías MJ, Fortún J, et al; COVID-19 ID Team: Description of COVID-19 in HIV-infected individuals: A single-centre, prospective cohort. Lancet HIV 7: e554-e564, 2020.

21. Agarwal N, Iyer D, Gabbi C, Saha P, Patel SG, Mo Q, Chang B, Goswami B, Schubert U, Kopp JB, et al: HIV-1 viral protein $\mathrm{R}$ (Vpr) induces fatty liver in mice via LXR $\alpha$ and PPAR $\alpha$ dysregulation: Implications for HIV-specific pathogenesis of NAFLD. Sci Rep 7: 7, 2017

22. Wanjalla CN, McDonnell WJ and Koethe JR: Adipose tissue T Cells in HIV/SIV infection. Front Immunol 9: 2730, 2018.

23. Alberca RW, Oliveira LM, Branco ACCC, Pereira NZ and Sato MN: Obesity as a risk factor for COVID-19: An overview. Crit Rev Food Sci Nutr: Jun 15, 2020 (Epub ahead of print).

24. Lukic L, Lalic NM, Rajkovic N, Jotic A, Lalic K, Milicic T, Seferovic JP, Macesic M and Gajovic JS: Hypertension in obese type 2 diabetes patients is associated with increases in insulin resistance and IL- 6 cytokine levels: Potential targets for an efficient preventive intervention. Int J Environ Res Public Health 11: 3586-3598, 2014.

25. Gervasoni C, Meraviglia P, Riva A, Giacomelli A, Oreni L, Minisci D, Atzori C, Ridolfo A and Cattaneo D: Clinical features and outcomes of patients with human immunodeficiency virus with COVID-19. Clin Infect Dis 71: 2276-2278, 2020.

26. Cooper TJ, Woodward BL, Alom S and Harky A: Coronavirus disease 2019 (COVID-19) outcomes in HIV/AIDS patients: A systematic review. HIV Med 21: 567-577, 2020.

This work is licensed under a Creative Commons Attribution-NonCommercial-NoDerivatives 4.0 International (CC BY-NC-ND 4.0) License. 